\title{
SYNCHROTRON RADIATION DIFFRACTION STUDIES OF MAGNETIC MATERIALS
}

\author{
TH. BRÜCKEL \\ Hamburger Synchrotron-Strahlungslabor HASYLAB \\ at Deutsches Elektronen-Synchrotron DESY \\ 22603 Hamburg, Germany
}

\begin{abstract}
Since the advent of high brilliance synchrotron radiation sources, magnetic X-ray diffraction has become a standard microscopic probe for the investigation of magnetic structures, magnetic phase transitions and magnetic disorder phenomena. Modern experiments highlight the complementarity of this new probe to the standard neutron diffraction technique by taking advantage of the high momentum space resolution, the element sensitivity, the possibility to separate spin- and orbital contributions etc. In this paper we present selected experiments performed at HASYLAB which exemplify the present possibilities of resonant and non-resonant magnetic $\mathrm{X}$-ray diffraction.
\end{abstract}

PACS numbers: $75.25 .+z, 75.40 .-\mathrm{s}$

\section{Introduction}

Most of our present knowledge of the atomic structure of condensed matter results from X-ray diffraction studies, which probe the interaction of the electric field with the electric charge of the electron. However, since X-rays represent an electromagnetic radiation and since in magnetic materials some electrons carry a magnetic moment due to spin- and angular momentum, we naturally would expect a magnetic interaction in addition to the pure charge interaction. Even so this interaction was well established in theory [1] since Klein-Nishina in 1929, the first magnetic diffraction effect was demonstrated only in 1972 by de Bergevin and Brunel [2] with a commercial X-ray tube. The same authors gave a classical picture of the interaction process, deduced the detailed polarization dependence and presented measurements on ferromagnetic compounds in a subsequent paper [3]. However, since the magnetic interaction gives just a relativistic correction to the cross-section, the amplitudes of magnetic diffraction are down by approximately three orders of magnitude as compared to charge scattering, resulting in an intensity ratio of about $10^{-6}$. Therefore magnetic X-ray scattering was considered an exotic topic until the experiments on Ho by Gibbs et al. [4], which took advantage of the high brilliance of a synchrotron radiation X-ray source thus compensating 
by a high photon flux at the sample position for the weak magnetic scattered intensities. The polarization properties and the tunability of synchrotron radiation offered new perspectives for magnetic $\mathrm{X}$-ray investigations. This was again demonstrated on Ho [5] by an attempt to separate spin- and angular momentum with polarization analysis and by the observation of a resonance enhancement of the magnetic signal at the absorption edges.

Nowadays, synchrotron radiation techniques for the study of properties of magnetic materials are well established. Very widespread is the application of incoherent probes which measure a macroscopic ensemble average of local magnetic properties. Among these we mention Kerr microscopy, measurements of the Faraday effect and the linear or circular X-ray magnetic dichroism. The Kerr- and Faraday effect measure the rotation of the plane of polarization of an electromagnetic wave as it is reflected from or transmitted through a magnetic material, respectively. Magnetic circular dichroism describes the difference in the absorption of right- and left circularly polarized X-rays by magnetic materials. It measures essentially the same quantities as the Kerr- and Faraday effect, namely the orbital and spin contributions to the magnetic moments with element and certain site characteristics. Kerr microscopy and X-ray topography are used for magnetic domain imaging. Absorption techniques become local microscopic probes when the spin resolved X-ray absorption fine structure is observed. In analogy to classical EXAFS experiments, such measurements provide information about the local environment, but are explicitly sensitive to the magnetic neighbours only. True microscopic spatial resolution is obtained with the coherent probes, namely magnetic X-ray diffraction (as well non-resonant as resonant exchange scattering) and nuclear resonant scattering. Magnetic scattering provides a wealth of information on magnetic correlation lengths, the local magnetic moments and environment, the magnetic structure and phase transitions. Magnetic reflectivity is the corresponding probe for the investigation of magnetic thin films. Nuclear resonant scattering yields information on hyperfine fields and might eventually become important for the measurement of magnetic excitations. Resonant diffraction and absorption techniques are intimately related by the optical theorem, which states that the attenuation coefficient is proportional to the imaginary part of the forward scattering amplitude. In this sense, diffraction experiments comprise absorption techniques, but in addition they provide true atomic resolution. In this impressive list of synchrotron radiation techniques we want to finally mention magnetic Compton scattering for the determination of the spin resolved electron momentum density and angular- and spin resolved photoemission, which gives the spin-resolved band structure.

Many of these topics are discussed in detail in a recent textbook [6]. In what follows we will concentrate on magnetic X-ray diffraction. We will introduce some basic concepts of non-resonant and resonant diffraction in Sec. 2 and present results of non-resonant diffraction at high photon energies in Sec. 3. Examples of resonance exchange scattering experiments performed at HASYLAB are given in Sec. 4. A summary of some important features of magnetic X-ray scattering is given in Sec. 5. 


\section{The cross-section for magnetic $\mathrm{X}$-ray diffraction}

The cross-section for non-resonant magnetic X-ray scattering has been discussed in various approximations by several authors. Here we follow a presentation given by Blume [7] and Blume and Gibbs [8] based on a non-relativistic treatment in second-order perturbation theory. At moderately high X-ray energies, the elastic cross-section for scatterring of photons with incident polarization $\varepsilon$ into a state of final polarization $\varepsilon^{\prime}$ can be written as

$$
\left.\frac{\mathrm{d} \sigma}{\mathrm{d} \Omega}\right|_{\varepsilon \rightarrow \varepsilon^{\prime}}=\left[\frac{e^{2}}{m c^{2}}\right]^{2}\left|\left\langle f_{\mathrm{C}}\right\rangle_{\varepsilon^{\prime} \varepsilon}+\mathrm{i} \frac{\lambda_{\mathrm{C}}}{d}\left\langle f_{\mathrm{M}}\right\rangle_{\varepsilon^{\prime} \varepsilon}\right|^{2}
$$

Here $r_{\mathrm{e}}=e^{2} / m c^{2} \approx 2.818 \mathrm{fm}$ denotes the classical electron radius, $\lambda_{\mathrm{C}}=$ $h / m c=2.426 \mathrm{pm}-$ the Compton length of an electron. The scattering amplitudes $\left\langle f_{\mathrm{C}}\right\rangle$ and $\left\langle f_{\mathrm{M}}\right\rangle$ are given as matrices which describe the polarization dependencies of charge and magnetic scattering, respectively. Here we discuss the case of linear polarization, described by unit vectors perpendicular to the wave vectors of incident and scattered photons, $k$ and $k^{\prime} . \sigma$-polarization corresponds to the basis vector perpendicular to the scattering plane, $\pi$-polarization corresponds to the vectors in the $k, k^{\prime}$ plane. The basis vectors for the components of the magnetic moment of the sample and for the polarization states are defined as follows:

$$
\begin{aligned}
& \widehat{u}_{1}=\left(k+k^{\prime}\right) /\left|k+k^{\prime}\right|, \quad \widehat{u}_{2}=\left(k^{\prime} \times k\right) /\left|k^{\prime} \times k\right| \equiv \sigma \equiv \sigma^{\prime}, \\
& \widehat{u}_{3}=\left(k^{\prime}-k\right) /\left|k^{\prime}-k\right|, \equiv Q / Q, \quad \pi=\widehat{k} \times \sigma, \quad \pi^{\prime}=\widehat{k}^{\prime} \times \sigma^{\prime} .
\end{aligned}
$$

In this basis the matrices in (1) can be written as $-\left\langle f_{M}\right\rangle$ for the magnetic part:

\begin{tabular}{c|cc} 
to \from & \multicolumn{1}{c}{$\pi$} \\
\hline$\sigma^{\prime}$ & $S_{2} \cos \theta$ & {$\left[\left(L_{1}+S_{1}\right) \cos \theta+S_{3} \sin \theta\right] \sin \theta$} \\
$\pi^{\prime}$ & {$\left[-\left(L_{1}+S_{1}\right) \cos \theta+S_{3} \sin \theta\right] \sin \theta$} & $\left(2 L_{2} \sin ^{2} \theta+S_{2}\right) \cos \theta$
\end{tabular}

$-\left\langle f_{\mathrm{C}}\right\rangle$ for charge scattering:

\begin{tabular}{c|cc} 
to $\backslash$ from & $\sigma$ & $\pi$ \\
\hline$\sigma^{\prime}$ & $\rho(\boldsymbol{Q})$ & 0 \\
$\pi^{\prime}$ & 0 & $\rho(\boldsymbol{Q})(\cos 2 \theta)$
\end{tabular}

Here $S_{i}=S_{i}(Q)$ and $L_{i}=L_{i}(Q)(i=1,2,3)$ denote the components of the Fourier transform of the magnetization density due to the spin and orbital angular momentum, respectively. $\rho(Q)$ denotes the Fourier transform of the electronic charge density distribution.

As can be seen from Eq. (1), magnetic scattering is a relativistic correction to charge scattering. For coherent elastic Bragg scattering, the ratio between the magnetic and the charge amplitude is determined by the momentum transfer and therefore we have written the pre-factor for the magnetic amplitude in the cross-section (1) as $\lambda_{C} / d$ which emphasises that for a given Bragg reflection the ratio between magnetic and charge scattering is virtually independent of photon 
energy, at least to within the approximations leading to (1). Equation (1) contains three terms: pure Thomson scattering, purely magnetic scattering and an interference term. The latter becomes important if charge- and magnetic scattering occur at the same position in reciprocal space, which is the case for ferromagnets. Equations (3) and (4) show that magnetic scattering can be discriminated from charge scattering by a polarization analysis experiment, where the off-diagonal terms $\sigma \rightarrow \pi^{\prime}$ or $\pi \rightarrow \sigma^{\prime}$ are being measured. Finally, Eq. (3) shows that the spin and orbital contributions have different angular- and polarization dependencies and can therefore be distinguished in principle.

For what follows it is of interest to examine the high energy limit of the purely magnetic diffraction cross-section. In this limit, the cross-section becomes virtually independent of polarization and is sensitive only to the component of the Fourier transform of the spin density distribution perpendicular to the scattering plane

$$
\left.\frac{\mathrm{d} \sigma}{\mathrm{d} \Omega}\right|_{\text {magnetic }}=r_{0}^{2}\left(\frac{\lambda_{\mathrm{C}}}{d}\right)^{2}\left|S_{2}(Q)\right|^{2} .
$$

Thus at high photon energies around $100 \mathrm{keV}$, the pure spin density distribution becomes accessible without polarization analysis, while in neutron diffraction always the sum $L+2 S$ is being measured.

If the X-ray energy is tuned to the absorption edge of magnetic elements, resonance phenomena occur due to second-order perturbation theory [9]. Electric multipole (predominantly dipole and quadrupole) operators induce virtual transitions between core levels and unoccupied states above the Fermi energy. These processes become sensitive to the magnetic state due to the difference in occupation of minority and majority bands leading to resonant exchange scattering [10] as illustrated schematically in Fig. 1. Intensity gain factors of typically 50 are obtained for the lanthanide $L_{\mathrm{II}}$ and $L_{\mathrm{III}}$ edges. At the $M_{\mathrm{IV}}$ edge of actinides this intensity gain can be as high as seven orders of magnitude [11]. Resonant diffraction should allow a spectroscopy of the electronic states above the Fermi edge and renders magnetic diffraction sensitive to the magnetic species.

Close to the absorption edges, an energy dependent amplitude has to be added to the expression (1) for the scattering cross-section. In dipole approximation, this amplitude reads

$$
f_{\text {res }}^{E 1}(E)=f_{0}(E)+f_{\text {circ }}(E)+f_{\text {lin }}(E),
$$

with

$$
\begin{gathered}
f_{0}(E)=\left(\varepsilon^{\prime} \cdot \varepsilon\right)\left[F_{+1}^{1}+F_{-1}^{1}\right], \quad f_{\text {circ }}(E)=\mathrm{i}\left(\varepsilon^{\prime} \times \varepsilon\right) \cdot m\left[F_{-1}^{1}-F_{+1}^{1}\right], \\
f_{\text {lin }}(E)=\left(\varepsilon^{\prime} \cdot m\right)(\varepsilon \cdot m)\left[2 F_{0}^{1}-F_{+1}^{1}-F_{-1}^{1}\right] .
\end{gathered}
$$

$f_{0}$ is independent of the magnetic state (i.e. the conventional anomalous charge scattering), while $f_{\text {circ }}$ and $f_{\text {lin }}$ are the amplitudes connected for the special case of forward scattering with circular and linear dichroism, respectively. All three amplitudes have different polarization properties. $f_{\text {circ }}$ depends linear on the magnetic moment $m$, while $f_{\text {lin }}$ depends quadratic on $m$. Therefore for antiferromagnets 


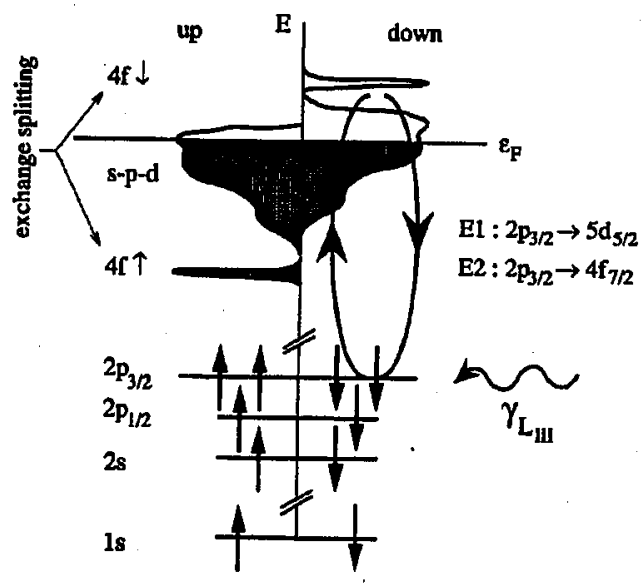

Fig. 1. Schematic diagram illustrating the effect of resonance exchange scattering at a lanthanide $L_{\text {III }}$ absorption edge. The sensitivity to the magnetic state results from the exchange splitting of the $4 f$ and the conduction band.

only $f_{\text {circ }}$ gives a contribution at positions in reciprocal space separated from the main charge reflections by the magnetic propagation vector. Finally, for a simple one level excitation, the energy dependence of the amplitudes is contained in the oscillator strengths

$$
F_{M}^{1}=\frac{\alpha_{\mathrm{M}}}{\left(\omega-\omega_{\text {res }}\right)-\mathrm{i} \Gamma / 2 \hbar} .
$$

Here $\omega$ denotes the photon energy, $\omega_{\text {res }}-$ the position of the absorption edge and $\Gamma-$ the resonance width. The phenomenological parameter $\alpha_{M}$ gives a measure for the amplitude of the resonance and stands for the product of the transition matrix elements.

\section{High energy non-resonant magnetic $\mathrm{X}$-ray diffraction}

Because of the resonance enhancement, most magnetic X-ray diffraction studies nowadays deal with resonant exchange scattering. However, for transition metal ions, only $K$-edges lie in the range of hard X-ray wavelengths, where atomic resolution is achievable. Due to the dipole and quadrupole selection rules and the small overlap between core $1 s$-states and the magnetic sensitive $3 d$ or $4 p$ energy bands, resonance enhancements are negligible at transition metal $K$-edges and one is left with neutron- or non-resonant magnetic X-ray scattering. Here we discuss a new method which might overcome these difficulties, namely the non-resonant magnetic scattering of very hard X-rays with energies above $80 \mathrm{keV}$ [12-14]. From (5) it follows that with high energy X-ray diffraction one can determine the spin density distribution independent of the polarization of the incident beam and without analysis of the final polarization after scattering. While in neutron diffraction only the total magnetic moment, proportional to the sum $L+2 S$ is accessible, $\mathrm{X}$-ray diffraction in the conventional energy range requires polarization analysis to separate the spin momentum $S(Q)$ from the orbital angular momentum density $\boldsymbol{L}(Q)$. 


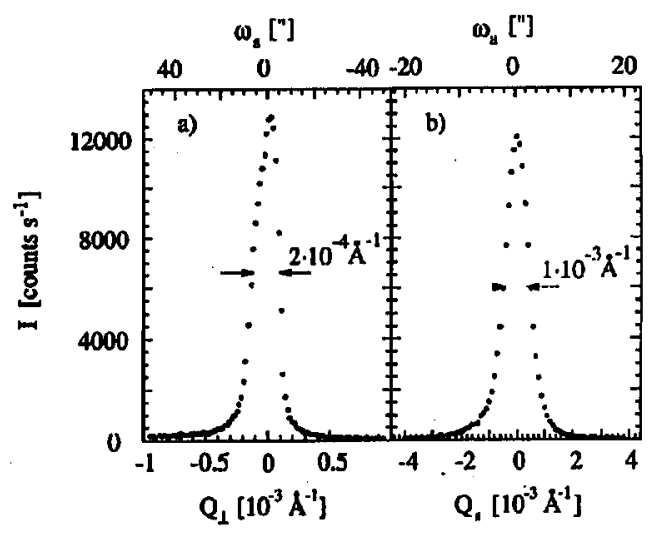

Fig. 2. Representative scans of the magnetic 300 -reflection of $\mathrm{MnF}_{2}$ at $T=5 \mathrm{~K}$. The $\mathrm{X}$-ray energy was $80 \mathrm{keV}$. Part (a) shows a transversal scan, part (b) a longitudinal scan taken in three-crystal mode with a Si 311 analyser.

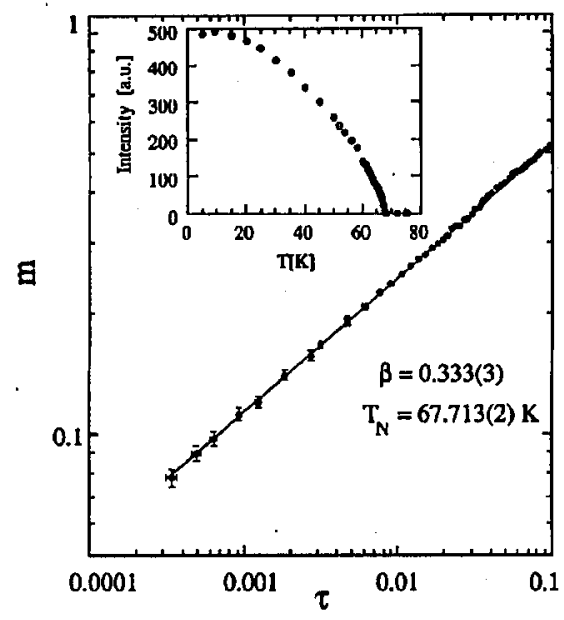

Fig. 3. Reduced magnetization versus reduced temperature close to the magnetic phase transition of $\mathrm{MnF}_{2}$ in a double logarithmic scale. The solid circles are experimental data points, the line represents a refinement according to (9). The inset shows the temperature dependence of the intensity of the magnetic 300 reflection in the expanded temperature range 5 to $80 \mathrm{~K}$.

The additional principal feature of high energy magnetic X-ray diffraction is the drastic increase in penetration depth. For $3 d$ transition metals, the absorption length $1 / \mu$ increases from some $\mu \mathrm{m}$ at $8 \mathrm{keV}$ to several $\mathrm{mm}$ at $80 \mathrm{keV}$. This leads to a volume enhancement of the signal which is only partly compensated by the $\lambda^{2}$ term for the reflectivity. Moreover, true bulk properties become accessible, a point especially important for studies of magnetic disorder phenomena. Neutron 
and photon experiments of bulk properties from the same crystal become possible, where one advantage of the $\mathrm{X}$-ray study is the high intrinsic resolution of about $10^{-3} \AA^{-1}$ longitudinal and $2 \times 10^{-4} \AA^{-1}$ transversal. For a detailed discussion of the general aspects of high energy synchrotron radiation experiments we refer to Schneider [15].

To test the potential of the new method we conducted at HASYLAB and ESRF experiments on the antiferromagnetic model system $\mathrm{MnF}_{2}$. The X-ray energy was $80 \mathrm{keV}$ and we used a three-crystal setup with annealed Si 311 crystals as monochromator and analyser. The scans shown in Fig. 2 demonstrate the high peak count rate of 12000 counts/second, the good peak-to-background ratio of 230:1 and the excellent $Q$ space resolution obtained for the magnetic $300 \mathrm{Bragg}$ reflection. By varying the path length of the beam through the crystal, the effect of volume enhancement of the intensity diffracted by magnetic reflections could be demonstrated. The $Q$ dependence of the magnetic and charge reflections has been measured and agrees well with theory. With a rotation around the scattering vector, the spin component $S_{2}$ can be made to zero and with it vanishes the magnetic scattering in full agreement to (5). Figure 3 shows a measurement of the temperature dependence of the sublattice magnetization. In the critical region close to the Néel temperature $T_{\mathrm{N}}$, the reduced sublattice magnetization $m=M(T) / M(T=0)$ follows very accurately a power law behaviour

$$
m(\tau)=D \tau^{\beta},
$$

as a function of the reduced temperature $\tau=\left(T_{\mathrm{N}}-T\right) / T_{\mathrm{N}}$. The value of the critical exponent is $\beta=0.333(3)$ with $T_{\mathrm{N}}=67.713(2) \mathrm{K}$.

\section{Resonant exchange scattering in transition metal and lanthanide compounds}

As an example of the effect of resonance exchange scattering, we show in Fig. 4 raw data taken at the W1 beam line at HASYLAB for the $L_{\text {II }}$ resonance of GdS. Due to the high absorption cross-section of Gd for thermal neutrons, no detailed neutron diffraction studies exist for GdS. With X-rays a comfortable count rate of about 3000 photons per second was obtained for the 9/2 1/2 1/2 reflection on resonance and we could verify the assumed type II antiferromagnetic ordering on the fcc lattice. While resonant exchange scattering can in principle give information about the density of unoccupied states above the Fermi level, most resonance line shapes can well be approximated with the simple two-level model of Eq. (8). This is also true for the resonance shown in Fig. 4, where only a small asymmetry remains after absorption correction.

One of the most structured resonances observed so far occurs at the $K$-edge of manganese in the perovskite type compound $\mathrm{RbMnF}_{3}$ [16]. We measured the integrated intensities of the magnetic $1 / 21 / 25 / 2$ Bragg peak at $20 \mathrm{~K}$ well below the Neel temperature $T_{\mathrm{N}}=54.5 \mathrm{~K}$ as a function of photon energies in the range 6.5 to $6.65 \mathrm{keV}$. At the absorption edge of $6.539 \mathrm{keV}$ a small resonance enhancement by a factor of roughly two was observed. A second resonance follows about $5.5 \mathrm{eV}$ above the first. Both resonances have a width of $4 \mathrm{eV}$. As a third feature a much weaker and broader enhancement occurs about $45 \mathrm{eV}$ above the edge. Possible 

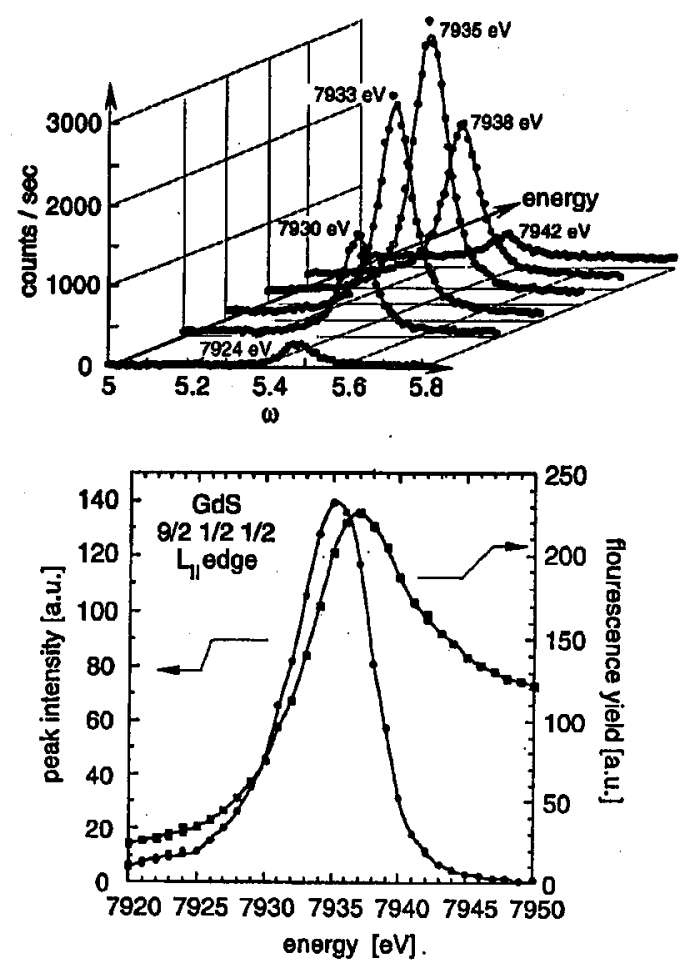

Fig. 4. The resonance enhancement of the magnetic signal at the $9 / 21 / 21 / 2$ superstructure peak of $\mathrm{GdS}$ at the $\mathrm{Gd} L_{\mathrm{II}}$ edge measured at $4.2 \mathrm{~K}$. The top diagram shows raw data of rocking curve scans at various photon energies. The bottom diagram shows as a function of photon energy the integrated intensities together with the structure of the absorption edge.

mechanisms for these resonance features are dipolar $(1 s-4 p)$ and quadrupolar $(1 s-3 d)$ transitions followed by a multiple excitation involving both the $1 s$ and $3 p$ inner shells.

An application of resonance exchange scattering lies in the investigation of the critical behaviour at magnetic phase transitions. Here the small cross-section for magnetic X-ray scattering eliminates extinction effects which can play a role for the Bragg component. Moreover, the high Q-space resolution permits a better separation of the critical Bragg- and diffuse component. We have utilized these features in an investigation of the critical behaviour of the sublattice magnetization of the antiferromagnets GdS and EuTe [17]. Due to the spherical symmetric ground state of the magnetic $\mathrm{Eu}^{2+}$ or $\mathrm{Gd}^{3+}$ ions, both compounds are expected to show an exponent $\beta$ of the sublattice magnetization typical of $3 d$ Heisenberg systems, i.e. $\beta=0.367$ (for a definition of $\beta$, see (9)). However, both GdS and EuTe crystallize in a rock salt structure, where the magnetic ions occupy sites on an fcc lattice, which results in a high degree of frustration in case of antiferromagnetic couplings. Such highly frustrated antiferromagnets are believed to belong 


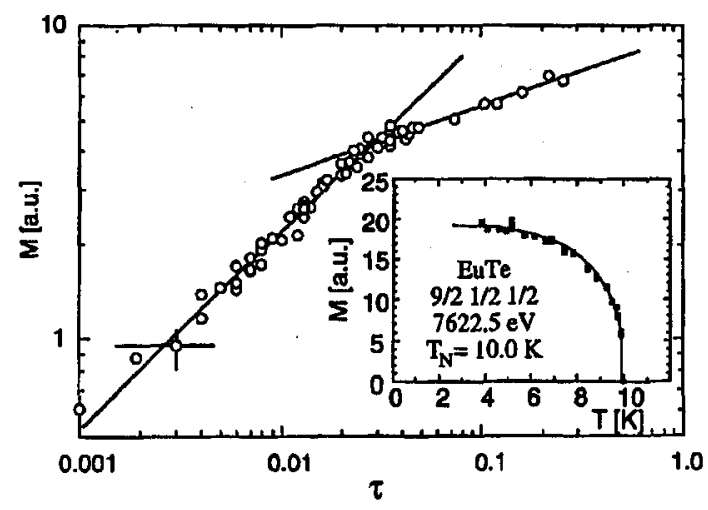

Fig. 5. Double logarithmic plot of the square root of the intensity of the magnetic 9/2 1/2 1/2 superstructure peak of EuTe versus reduced temperature as defined in (9). The data were taken with the X-ray energy tuned to the Eu $L_{\mathrm{II}}$ resonance. The inset shows a measurement over a wider temperature range.

to new universality classes. Indeed, with resonant magnetic X-ray scattering we found marked deviations from the expected critical exponent for both compounds. Figure 5 shows the data for EuTe. We were able to approach the phase transition at $T_{\mathrm{N}}=10.0 \mathrm{~K}$ as close as $0.01 \mathrm{~K}$ with a stability in the $\mathrm{mK}$ range. Our data clearly indicate a crossover behaviour from a critical exponent $\beta=0.28(1)$ observed earlier with neutron diffraction [18] to a much larger exponent $\beta>0.6$ very close to $T_{\mathrm{N}}$.

\section{Summary}

The above examples clearly demonstrate that X-ray diffraction from magnetic materials has become a microscopic probe of magnetism, complementary to the traditional probe of neutron scattering. First, it allows the investigation of strongly neutron-absorbing materials (compare our measurements of GdS and EuTe). Second, it stands out by the high momentum-space resolution of typically $10^{-4} \AA^{-1}$, about one to two orders of magnitude better than a standard neutron experiment. This is of advantage for the investigation of incommensurate structures, magnetic disorder phenomena which involve a broadening of the magnetic Bragg reflection or critical scattering. In critical scattering investigations of the sublattice magnetization, the magnetic Bragg peak can be well separated from critical diffuse scattering (see our examples of EuTe and $\mathrm{MnF}_{2}$ ). For measurements of the critical diffuse scattering, the high $Q$-space resolution permits a detailed line shape analysis and a closer approach of the phase transition. Moreover, due to the high incident energy the full integration over the energy spectrum of magnetic fluctuations is guaranteed. Third, X-ray diffraction is a perfect tool for the investigation of near-surface phenomena and ultimately of surface magnetism.

Considering non-resonant and resonant scattering, some specific applications are opened for each technique. In the case of resonance-exchange scattering, most evidently the resonance-enhancements at the absorption edges allow the investigation of samples with very small magnetic moments. In principle a "magnetic" 
spectroscopy of the unoccupied levels above the Fermi edge should be possible (compare our discussion of the results for $\mathrm{GdS}$ and $\mathrm{RbMnF}_{3}$ ). Finally the resonance effects render magnetic scattering element specific. In alloys and mixed crystals it is now possible to investigate the magnetic order of the various magnetic elements separately. The big drawback of resonant scattering is, however, that as for any second-order perturbation process the scattered intensity is not easily interpretable in a simple and fundamental quantity such as the magnetic moment. This is one reason why non-resonant scattering remains essential. It permits the separate determination of the spin- and angular moment contributions to the form factor. The rich cross-section of non-resonant scattering gives complementary information to neutron diffraction in the case of complicated magnetic structures. As a special case of non-resonant scattering we introduced the diffraction of high-energy X-rays with energies around or above $100 \mathrm{keV}$. The large penetration power makes this radiation a true volume probe just like neutron scattering. A simple sample environment without specific X-ray transparent windows can be used and X-ray and neutron diffraction experiments can be performed from the same bulk crystal. A volume enhancement of the signal is obtained, independent of composition. The short wavelength and the small cross-section eliminate extinction effects, which allows precision measurements of structure factors within the first Born approximation. Finally, the simple form of the cross-section (5) makes possible a mapping of the spin momentum distribution alone without polarization analysis.

\section{Acknowledgments}

This work was funded by the Bundesministerium für Bildung und Forschung (BMBF), project number 03-BR4DES-2 and the European Community under the Human Capital and Mobility Initiative, contract number ERBCHRXCT930135. The presented experiments were performed within larger collaborations, including the HASYLAB scientists J. Strempfer, U. Rütt and J.R. Schneider and ESRF colleagues K.D. Liss and $\mathrm{T}$. Tschentscher for the experiments on $\mathrm{MnF}_{2}$, the ESRF colleagues A. Stunault, F. de Bergevin, D. Wermeille and C. Vettier for the study of $\mathrm{RbMnF}_{3}, \mathrm{~K}$. Mattenberger, ETH Zürich for GdS and U. Köbler from KFA Jülich for EuTe.

\section{References}

[1] P.M. Platzman, N. Tzoar, Phys. Rev. B 9, 3556 (1970).

[2] F. de Bergevin, M. Brunel, Phys. Lett. A 39, 141 (1972).

[3] F. de Bergevin, M. Brunel, Acta Crystallogr. A 37, 314 (1981).

[4] D. Gibbs, D.E. Moncton, K.L. D'Amico, J. Bohr, B.H. Grier, Phys. Rev. Lett. 55, 234 (1985).

[5] D. Gibbs, D.R. Harshman, E.D. Isaacs, D.B. McWhan, D. Mills, C. Vettier, Phys. Rev. Lett. 61, 1241 (1988).

[6] S.W. Lovesey, S.P. Collins, X-Ray Scattering and Absorption by Magnetic Materials, Clarendon Press, Oxford 1996.

[7] M. Blume, J. Appl. Phys. 57, 3615 (1985).

[8] M. Blume, D. Gibbs, Phys. Rev. B 37, 1779 (1988). 
[9] M. Blume, in: Resonant Anomalous X-Ray Scattering, Eds. G. Materlik, C.J. Sparks, K. Fischer, North-Holland, Amsterdam 1994, p. 495.

[10] J.P. Hannon, G.T. Trammell, M. Blume, D. Gibbs, Phys. Rev. Lett. 61, 1245 (1988) and Erratum: Phys. Rev. Lett. 62, 2644 (1989).

[11] E.D. Isaacs, D.B. McWhan, C. Peters, G.E. Ice, D.P. Siddons, J.B. Hastings, C. Vettier, O. Vogt, Phys. Rev. Lett. 62, 1671 (1989).

[12] Th. Brückel, M. Lippert, R. Bouchard, T. Schmidt, J.R. Schneider, W. Jauch, Acta Crystallogr. A 49, 679 (1993).

[13] M. Lippert, Th. Brückel, Th. Köhler, J.R. Schneider, Europhys. Lett. 27, 537 (1994).

[14] J. Strempfer, Th. Brückel, U. Rütt, J.R. Schneider, K.D. Liss, T. Tschentscher, Acta Crystallogr. A 52, 438 (1996).

[15] J.R. Schneider, Synchrotron Radiat. News 8, 26 (1995).

[16] A. Stunault, F. de Bergevin, D. Wermeille, Th. Brückel, C. Vettier, HASYLAB annual report 1994, p. 557.

[17] U. Köbler, Th. Brückel, HASYLAB annual report 1995, p. II-349.

[18] U. Köbler, I. Apfelstedt, K. Fischer, W. Zinn, E. Scheer, J. Wosnitza, H.v. Löhneysen, Th. Brückel, Z. Phys. B 92, 475 (1993). 